\title{
A Randomized Controlled Trial of Tissue Adhesive Versus Sutures in the Closure of Port-Site Incisions in Laparoscopic Surgery
}

\author{
Maniar N, Deshpande AA. \\ Department Of Surgery, Seth G.S. Medical College \& K.E.M. Hospital,Maharashtra University Of Health \\ Sciences, India
}

\begin{abstract}
Cyanoacrylates have been recognized since before the 1960s as excellent tissue adhesives. They are easy to use, cost-effective, and cosmetically favorable. There are no studies from India reporting the use of tissue adhesives in laparoscopic surgery. We carried out a prospective, interventional, randomized trial of non-absorbable simple interrupted sutures versus 2-octyl cyanoacrylate in the closure of skin incisions in laparoscopic surgery with the primary objective of assessing the efficacy (approximation achieved) of both methods. The secondary objectives were to compare the operative time for closure, post-operative pain, infection and cost. There were 20 participants, 10 in either arm. Consenting patients undergoing elective laparoscopic surgery in a single surgical unit were included. Descriptive statistics were used on demographic data. Comparisons between the two methods were made on the basis of Southampton Wound Assessment Scale-SWAS grade for healing, Visual Analogue Scale-VAS scores for pain, intra-operative time, and cost. Apart from cost which was significantly higher for adhesive group, the groups did not differ significantly in terms of pain, wound approximation, and infection. Octyl cyanoacrylate is comparable to non-absorbable simple interrupted sutures in terms of closure time, post-operative pain, wound approximation and infection. The cost of using octyl cyanoacrylate is substantially higher than traditional suture material.
\end{abstract}

Keywords: Cyanoacrylate, Laparoscopic port site closure, tissue adhesive

\section{Introduction}

A number of techniques are at the surgeon's disposal today for the closure of skin incisions like sutures, staples, adhesive tape, skin glue etc. The large array of new materials makes the proper choice for closure a challenge [1]. The choice of a particular method is based primarily on the surgeon's experience.

Cyanoacrylates have been recognized since before the 1960s as excellent tissue adhesives [2]. They are easy to use, cost-effective, and cosmetically favourable [3]. Abundant literature is already available with regards to its application in open surgery $[4,5,6]$.

There are a few studies from different countries reporting the application of tissue adhesive in laparoscopic surgery. Sebesta et al show that Laparoscopic port-site skin closure with octylcyanoacrylate is rapid and effective [1]. A similar study from the UK shows that tissue adhesive for laparoscopic port site closure offers potential savings with respect to time and has comparable wound complication rates and cosmetic outcomes when compared with non-absorbable monofilament sutures [7]. A Polish study has also suggested the usefulness of the adhesive in good wound closure and correct healing [8]. Despite several reports, a meta-analysis of skin adhesives suggests that a multicentre randomized controlled trial is required in order to obtain stronger evidence [9].

There are no studies from India reporting the use of tissue adhesives in laparoscopic surgery. The suitability of its use in resource limited country and a different patient population could well be different from that elsewhere. Thus we carried out a randomized trial of non-absorbable simple interrupted sutures versus 2octyl cyanoacrylate in the closure of skin incisions in laparoscopic surgery with the primary objective of assessing the efficacy (approximation achieved) of both methods. The secondary objectives were to compare the operative time for closure, post-operative pain, infection and cost.

\subsection{Materials:}

\section{Materials And Methods}

1) High viscosity DERMABOND ${ }^{\mathrm{TM}}$ Topical Skin Adhesive manufactured by ETHICON, Sommerville, New Jersey.

2) ETHILON 3-0 ${ }^{\mathrm{TM}}$ Non-absorbable monofilament polyamide suture with sterilized $26 \mathrm{~mm} 3 / 8$ circle reverse cutting needle manufactured by JOHNSON \& JOHNSON LTD, Baddi, HP, India. 


\subsection{Methods:}

\subsubsection{Ethics}

The study was initiated after obtaining the approval of The Institutional Ethics Committee as per Ethical Guidelines for Biomedical Research on human subjects, Indian Council of Medical Research, New Delhi, 2006. Written informed consent was taken from all participants or their legally accepted representatives.

\subsubsection{Setting}

The study was carried out in the operation theatre of a general surgical unit of a tertiary-care urban teaching hospital

\subsubsection{Trial design}

It was a prospective, interventional randomized controlled study including 20 participants, 10 in either arm.

\subsubsection{Patients}

Over a period of two months, consenting patients undergoing elective laparoscopic surgery in one unit of the surgery department of a tertiary-care urban teaching hospital were selected in the study. They were enrolled after obtaining written informed consent from the participants or their legally acceptable representatives.

Inclusion Criteria

Patients:

1. Of either sex.

2. $\geq 18$ years of age.

3. Undergoing elective laparoscopic procedures.

Withdrawal Criteria: Patients who were converted to open surgery.

\subsubsection{Randomization and allocation concealment}

The randomization sequence was generated form www.randomization.com. No restrictions were applied. Allocation concealment was carried out using sequentially numbered opaque sealed envelopes.

Randomization, enrollment and patient assignment were carried out by NM.

\subsubsection{Sample size}

No formal calculations for sample size were made. Twenty patients enrolled in the study.

\subsubsection{Statistical analysis}

Descriptive statistics were used on demographic data. Comparisons between the two methods were made on the basis of Southampton Wound Assessment Scale-SWAS grade, Visual Analogue Scale-VAS scores, intra-operative time, and cost. The t-test was used to compare the intra-operative time and VAS scores across the two groups. The Fisher's exact test was used to compare the SWAS grades. Statistical analysis was carried out using the 'Graph pad Quick calc' online statistical calculator.

\subsubsection{Study procedure}

The study was conducted after obtaining approval from the institutional ethics committee. This was a prospective, interventional, randomized, consecutive, 2 month long study in the operation theatre of the general surgery department of a tertiary hospital. After obtaining written informed consent, the relevant clinical data were collected.

\section{The patients were then randomized into 2 arms;}

The intervention arm receiving DERMABOND ${ }^{\mathrm{TM}}$ (2-octyl cyanoacrylate) and the control arm receiving non-absorbable simple interrupted sutures with ETHILON 3-0 ${ }^{\mathrm{TM}}$ (non-absorbable monofilament polyamide suture).

Time taken for closure of the skin incision was recorded in seconds.

After the procedure, pain at the incision site as assessed by the patient on a visual analogue scale and wound approximation was checked on post- operative day 1.

Patients were examined once again on the day of suture removal i.e. at the end of 1 week for any signs of wound separation or dehiscence.

Both materials were available free of cost to patients. The cost of both materials was calculated based on data collected from the hospital pharmacy. 


\subsubsection{Outcome measures}

The following outcome measures were studied:

- Intra- operative time in seconds for closure of the skin incision

- VAS score for pain on post-operative day 1

- Southampton Wound Assessment Scale (SWAS) score for wound infection at the end of 1 week

- Cost

\section{Participant flow:}

\section{Results}

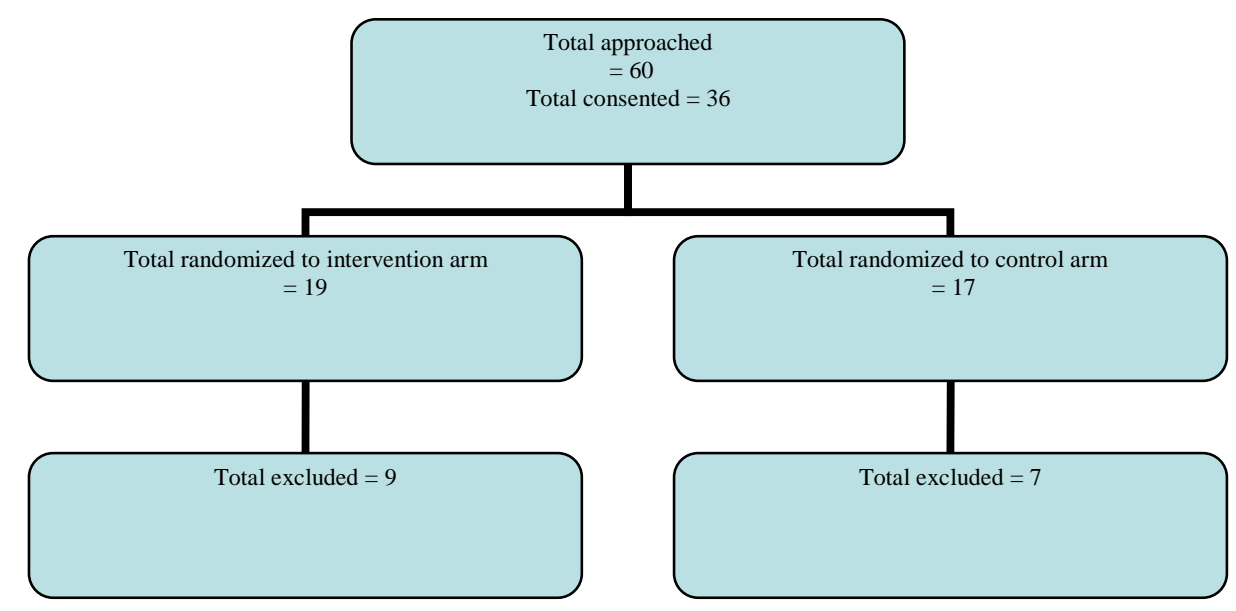

The reason for exclusion after randomization in all 16 excluded participants was failure to follow up. Reason for termination of trial: completion of sample size.

\subsection{Demographic Data:}

During the study period a total of 20 patients were enrolled; 10 in the intervention arm and 10 in the control arm. Thirteen $(65 \%)$ of the patients included in the study were females. The mean age of patients was $41 \pm 13$ years. The most commonly performed surgery was laparoscopic cholecystectomy $\{12(60 \%)\}$. Three patients $(15 \%)$ had an associated co-morbidity in the form of diabetes mellitus.

\subsection{Outcomes:}

\subsubsection{Wound approximation}

None of the wounds in either group had separated on post-operative day 1 . Thus the approximation was considered equal and adequate across both groups on day 1 post-op. On day 7, 4 patients in the adhesive group and 2 patients in the suture group experienced wound separation.

\subsubsection{Time for skin closure}

The comparison between times for closure (in seconds) across the two groups is shown below.

Table 1: Comparison between times for closure

\begin{tabular}{|l|l|l|}
\hline Group & Adhesive & Suture \\
\hline Mean & 198.40 & 171.10 \\
\hline SD & 62.96 & 29.03 \\
\hline N & 10 & 10 \\
\hline
\end{tabular}

Using the unpaired t-test, $p=0.229$.

\subsubsection{VAS score}

The comparison VAS scores (in mm) as recorded on day 1 post-operatively across the two groups is as follows:

Table 2: Comparison of VAS scores

\begin{tabular}{|l|l|l|}
\hline Group & Adhesive & Suture \\
\hline Mean & 27.60 & 35.20 \\
\hline SD & 22.27 & 19.32 \\
\hline N & 10 & 10 \\
\hline
\end{tabular}

On applying the unpaired t-test, $p=0.425$ 


\subsubsection{Infection}

On day 7 , the results were as follows:

In the group receiving the adhesive, 4 patients had normal wound healing (grade A) and the other 2 had minor complications (grade B) and 4 had infected wounds (grade C). In the group receiving sutures, 6 patients had a normal wound healing, 2 patients had minor complications and 2 had infected wounds.

Table 3: Comparison of SWAS grades

\begin{tabular}{|l|l|l|}
\hline \multicolumn{2}{|l|}{ SWAS grade (no. of patients) } \\
\hline Group & A or B & C \\
\hline Adhesive & 6 & 4 \\
\hline Suture & 8 & 2 \\
\hline
\end{tabular}

On applying the Fisher's exact test, $p=0.628$.

\subsubsection{Cost}

1) One unit Of Dermabond (octylcyanoacrylate) costs Rs. 911.00

2) One packet of Ethilon (non-absorbable polyamide suture) costs Rs. 113.00

Thus, Dermabond is nearly 8 times the cost of the suture. The difference in price is that of Rs. 798 per patient.

\section{Discussion}

In the immediate post-operative period, the approximation was equal and adequate in both groups. On the day 7 post-op, the intervention group fared better than the control in terms of approximation.

With regard to time required for closure, the control group fared better than the adhesive with a mean closure time of 171.1 seconds. The greater experience of surgeons with simple interrupted sutures as compared to tissue adhesive could explain this outcome. The difference in the mean closure time however was only 27.3 seconds, not statistically significant. This is in contrast to results from foreign countries which regularly use subcuticular sutures for skin closure and have reported time savings to the tune of 9 minutes per case with the use of adhesive [1,4].

In terms of post operative pain, the differences in VAS scores are not significant $(\mathrm{p}=0.42)$. When applied correctly, i.e. without seepage into the wound, the adhesive causes much smaller amounts of foreign body in the subcutaneous plane in contrast to suture lines which pass through it [7]. Hence, better pain outcomes were expected with the adhesive than the suture.

Four patients in the intervention group and 2 patients in the control group had infected wounds. While no statistical difference was found, fewer infections occurred in the control group. This is in disagreement to the fact that the adhesive is known to have anti-microbial effects against gram-positive organisms [5].

The cost of the suture material is substantially lower than the adhesive. Studies from the UK and USA have demonstrated that usage of tissue adhesives reduces costs by decreasing the operative time [4, 5]. A study from Texas has reported mean savings of $\$ 362$ with the usage of tissue adhesive as an alternative to subcuticular sutures [1]. In our setting, where non-absorbable simple interrupted sutures are the norm, and where accurate data on operating room costs is not available, such calculations are not feasible. A mean saving of 27 seconds is unlikely to cause enough savings to compensate for the high cost of the material even if such calculations are made.

The study was limited by its small sample size and that the intervention was not always performed by the same surgeon.

\section{Conclusion}

In conclusion, Octyl cyanoacrylate is comparable to non-absorbable simple interrupted sutures in terms of closure time, post-operative pain, wound approximation and infection. In our setting, the cost of using octyl cyanoacrylate is substantially higher than traditional suture material.

\section{Other Trial Information}

This trial was registered with the Clinical Trials Registry of India (CTRI)

- Reg no. CTRI/2012/11/003124.

\section{Acknowledgement}

We wish to thank Dr. A.N. Supe, Director (ME \& MH) and Dean (Seth G.S.Medical College \& K.E.M. Hospital), for permitting us to publish hospital data.

\section{References}

[1]. Sebesta MJ, Bishoff JT. Octylcyanoacrylate skin closure in laparoscopy.JSLS. 2004 Jan-Mar;8(1):9-14. 
[2]. Coover HN, Joyner FB, Sheerer NH, Wicker TH. Chemistry and performance of cyanoacrylate adhesive. Special Technical Papers. 1959;5:413-417.

[3]. Osmond MH, Klassen TP, Quinn JV. Economic evaluation comparing a tissue adhesive with suturing in the repair of pediatric facial lacerations. J Pediatr. 1995;126:892-895.

[4]. Krishnamoorthy B, Najam O, Khan UA, Waterworth P, Fildes JE, Yonan N. Randomized prospective study comparing conventional subcuticular skin closure with Dermabond skin glue after saphenous vein harvesting. Ann Thorac Surg. 2009 Nov;88(5):1445-9.

[5]. Brown JK, Campbell BT, Drongowski RA, Alderman AK, Geiger JD, Teitelbaum DH, Quinn J, Coran AG, Hirschl RB. A prospective, randomized comparison of skin adhesive and subcuticular suture for closure of pediatric hernia incisions: cost and cosmetic considerations.J Pediatr Surg. 2009 Jul;44(7):1418-22.

[6]. Handschel JG, Depprich RA, Dirksen D, Runte C, Zimmermann A, Kübler NR. A prospective comparison of octyl-2cyanoacrylate and suture in standardized facial wounds. Int J Oral Maxillofac Surg. 2006 Apr;35(4):318-23. Epub 2005 Dec 20.

[7]. Dowson CC, Gilliam AD, Speake WJ, Lobo DN, Beckingham IJ. A prospective, randomized controlled trial comparing n-butyl cyanoacrylate tissue adhesive (LiquiBand) with sutures for skin closure after laparoscopic general surgical procedures. Surg Laparosc Endosc Percutan Tech. 2006 Jun;16(3):146-50.

[8]. Szyłł K, Włodarczyk B, Krekora M, Lewy J, Oszukowski P, Wilczyński J. [Application of skin glue in wound repair after laparoscopy] .Ginekol Pol. 2002 Jun;73(6):567-70

[9]. Sajid MS, Siddiqui MR, Khan MA, Baig MK. Meta-analysis of skin adhesives versus sutures in closure of laparoscopic port-site wounds. Surg Endosc. 2009 Jun;23(6):1191-7. Epub 2009 Mar 